\title{
Kapitel 16: Epilogoi (Schlußtopik)
}

Zunächst ist vorauszuschicken, daß nicht alle byzantinischen Heiligenviten und nicht einmal alle Heiligenviten, die über ein Proöm verfügen, einen Epilog aufweisen können ${ }^{1}$. Offenbar wurde der Epilog in geringerem Maße für rhetorisch notwendig erachtet als das Proöm² .

Die Epiloge der byzantinischen Heiligenviten bilden das rhetorische Pendant zu den Prooimia. Proöm und Epilog rahmen die eigentliche Narratio der Vita ein. Dies entspricht der rhetorischen Gliederung in Vorwort, Hauptteil und Schluß. Die Epiloge lehnen sich dabei im Hinblick auf Form und Inhalt grundsätzlich stark an die Prooimia $\mathrm{an}^{3}$, sind jedoch zum einen häufig kürzer

1 Ein Beispiel dafür wäre etwa die Vita Niceph. Medic. (BHG 2297), allerdings könnte hier auch ein unfertiger Zustand einer Heiligenvita vorliegen: Bios kai Politeia (mit Proöm, aber ohne Miracula und Epilog: $\$ 1-19)$ - ein kleiner Hymnos $(\$ 20)$ - drei Miracula $(\$ 21-23)$ - ein Gebet an den hl. Vater ( $S$ 24). Man könnte sich vorstellen, daß das Abschlußgebet noch eine Weiterentwicklung zu einem Epilog, mit dem Schwerpunkt der Invokation, wie dies recht häufig vor-

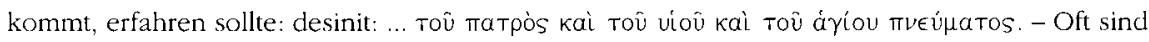
jedoch auch mechanische Beschädigungen der Hs. der Grund für das Fehlen eines Epilogs: Vita Nicephori Milesii (BHG 1338): Textabbruch; Vita Greg. Agrig. (BHG 707): Textabbruch, vgl Berger, Gregorios 23. 93. 127; Vita Euthymii patr. (BHG 651): Textabbruch, vgl. P. Karlin-Hayter, in: Vita Euthymii patr. (BHG 651) 30-32; Vita Phantini iun. (BHG 2366z): Textabbruch, vgl. E. Follieri, in: Vita Phantini iun. (BHG 2366z) 11. - Gelegentlich leiten Gebetsformeln vom Ende der Narratio, etwa der Bestattung, zum abschließenden „Amen" hinüber: Vita Nili iun. (BHG 1370) cap. 100, p. 135.

2 In einer Untersuchung zur Überlieferung der Vita des Niketas von Medikion (BHG 1341) hat Jan Olof Rosenqvist unlängst zwei Dinge hinsichtlich des in der einzigen griechischen $\mathrm{Hs}$. (Cod. Vat. gr. 1660) fehlenden Epilogs dieser Vita aufgezeigt: 1. Ein Epilog wurde von den byzantinischen Hagiographen zwar als rhetorisch notwendig, aber inhaltich am ehesten entbehrlich empfunden, so daß er bei notwendig werdenden Kürzungen als erstes gestrichen werden konnte, wie dies nach Ansicht von Rosenqvist im Falle der Vita des Niketas geschah. 2. Die Hagiographen hatten offenbar keine große Scheu, den Epilog einer früheren Vita fast wortwörtlich, mit nur wenigen Modifikationen in die von ihnen verfaßte Vita zu übernehmen, wie anscheinend der Epilog der Vita des Niketas von dem Mönch Petros in seine Version der Vita des Ioannikios (BHG 936) übernommen wurde. Vgl. Rosenqvist, Niketas 59-72.

3 Algemein gelten auch hier die bereits oben, in dem Kapitel zu den Prooimia, zu den jeweiligen Topoi angebrachten Bemerkungen. 
und weniger ausgearbeitet, zum anderen - besonders in der Invokation des Heiligen - stärker mit Elementen der Predigt angereichert. Folglich finden sich in den Epilogen zwar nicht sämtliche, aber doch zahlreiche Bestandteile der Topik der Prooimia wieder, allerdings sind diese Topoi häufig stark verknappt und oft nur angedeutet ${ }^{4}$.

Wir finden in den Epilogen 1. den Bescheidenheitstopos (topos modestiae), 2. den Begründungstopos (topos causae scribendi), 3. bestimmte Formeln (formulae) und 4. die Anrufung (invocatio). Dabei kommt in den Epilogen dem Bescheidenheitstopos und dem Anrufungstopos die größte Bedeutung zu.

\section{$\S 1$. Bescheidenheitstopos (topos modestiae)}

\section{A. Allgemein}

In der älteren Vita des Styliten Alypios (BHG 65) sagt der Verfasser eingangs des Epilogs über sich ${ }^{5}$ : „Und ich nun, der sehr unbedeutende und späte Nebenschößling deiner Pflanzung, Vater, erkühnte mich, dir diese Leichenrede darzubringen, ..."

Der Epilog der Vita des Gregorios Dekapolites (BHG 711) eröffnet mit dem allgemeinen Bescheidenheitstopos ${ }^{6}$ : „Dies alles nun ist mit unserer schwerfälligen Zunge und den knappen Mitteln des Verstandes voller Wagemut gesagt, nur um es denen, die Gott lieben, im Gedächtnis zu halten; ..." Etwas weiter unten heißt es ${ }^{7}$ : „Obwohl ich aber schwerfällig bin und keineswegs würdig, eine Lobrede zu halten, ..."

In der Vita des Nikon „Metanoeite“ (BHG 1366-67) behauptet der Verfasser ${ }^{8}$, er besitze nicht die Zunge, die sich zu derartigen Erzählungen und Wundern erheben könnte.

Auch in der Vita des Patriarchen Nikephoros (BHG 1335) enthält sich der Verfasser, Ignatios Diakonos, gegen Ende des Epilogs nicht einiger Bemerkun-

4 Vgl. auch Arbusow, Colores rhetorici $106 \mathrm{f}$.

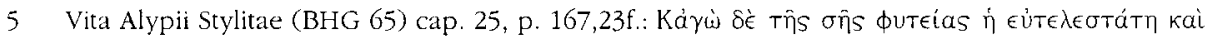

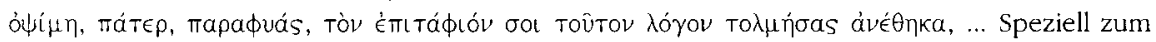
Topos modestiae im Epilog: Krueger, Hagiography 223-227.

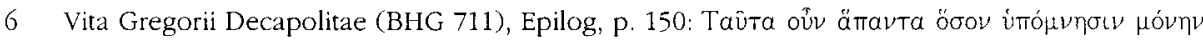

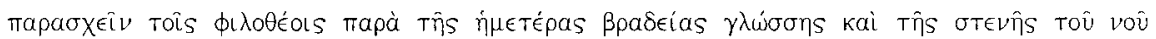

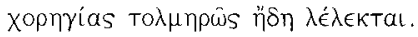

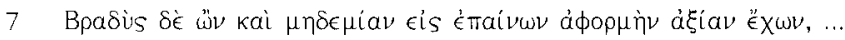

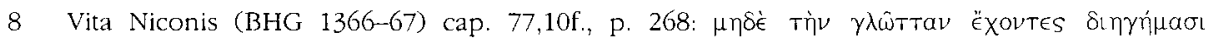

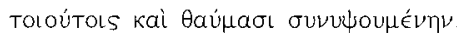


gen zu seiner angeblichen Unfähigkeit": „Du aber, o würdige und engelgleiche Seele, nimm unseren Eifer entgegen, der ich, im Vertrauen auf deinen Unterricht, die Aufgabe übernommen habe, die über meine Kräfte geht! ${ }^{10}$ Gewähre Nachsicht und Mitgefühl für die Torheit der Rede, in dem Wissen, daß das Gelingen nicht auf Lob hoffen darf, das Versagen aber auf milde Nachsicht!"

Im Epilog der älteren Version der Vita des Ioannikios (BHG 936; Petros) ${ }^{11}$ bittet der Verfasser, der Mönch Petros, im Rahmen der Invokation den Heiligen, von ihm, seinem nutzlosen Diener, die sehr kurze ${ }^{12}$ Grabrede entgegenzunehmen, die zwar unwürdig sei, aber doch im Glauben geschrieben ${ }^{13}$.

\section{B. Auftragstopos}

Im Schlußabschnitt der Vita des Maximos Homologetes (BHG 1234) bekennt der Verfasser ${ }^{14}$ : „Ganz und gar Urheber und Anlaß der Rede aber ist der von Dir (sc. Maximos) abhängige Hierarch, der mit dem gleichen Namen und der gleichen Art wie der Hirte der Herde von Myra geschmückt ist." Er wurde also von einem Bischof Nikolaos mit der Abfassung der Vita des Maximos beauftragt ${ }^{15}$.

In einer rhetorischen Passage am Schluß der Vita Tarasii (BHG 1698) wird vom Verfasser, Ignatios Diakonos, scheinbar ein Auftraggeber angesprochen ${ }^{16}$. Im Rahmen des Auftragstopos wird vom Verfasser behauptet, der Angesprochene habe Ignatios zur Abfassung der Vita, gegen dessen Willen, veranlaßt. Man hat in der Forschung vermutet ${ }^{17}$, daß sich hinter dem Angesprochenen Methodios I. verberge, der Patriarch von Konstantinopel zwischen 843 und 847. Doch bleibt die Anrede unpersönlich und aufgrund der Ausdrucksweise

9 Vita Nicephori patriarchae (BHG 1335) p. 217,31-36; vgl. dazu E. A. Fisher, in: Byzantine Defenders 142 Anm. 547.

$10 \mathrm{Vgl}$. die Emendation von E. A. Fisher, in: Byzantine Defenders 142 Anm. 546.

11 Vita Ioannicii (BHG 936; Petros) cap. 72, p. 435C.

12 Vgl. dazu D. F. Sullivan, in: Byzantine Defenders 350 Anm. 588.

13 Weitere Beispiele: Vita Athanasiae Aegin. (BHG 180) cap. 19, p. 194; Vita Macarii Pelecetae (BHG 1003) cap. 23, p. 163,19-26; Vita Georg. Amastr. (BHG 668) cap. 48, p. 70,15 - 71,1; Vita Steph. Sabait. (BHG 1670) cap. 185, p. 583D-E; Vita Stephani iun. (BHG 1666) cap. 77, p. $176,1 \mathrm{f}$.

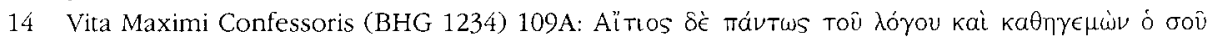

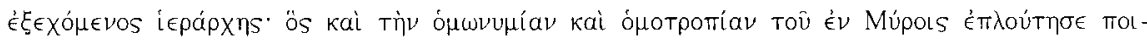
$\mu \in v$ ápхou.

15 Vgl. auch Lackner, Maximosvita 289.

16 Vita Tarasii (BHG 1698) cap. 70, p. $167,1-168,23$.

17 Vgl. beispielsweise Speck, Renaissance 569; s. dazu aber noch die Bemerkungen unten S. $397 \mathrm{f}$. in dieser Arbeit. 


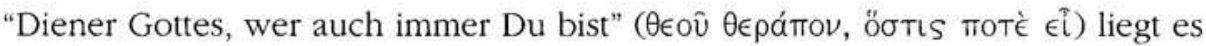
nahe, daß hier ganz allgemein der zukünftige Leser angesprochen wird. Es ist allerdings nicht auszuschließen, daß der Verfasser ungeachtet der allgemeinen Anrede mit seinem Schlußwort auf eine konkrete Person (vielleicht sogar den Patriarchen) zielte. Aufgrund fehlender Beweise bleibt dies jedoch reine Hypothese.

Im Epilog der Vita des Gregorios Dekapolites (BHG 711) heißt es ${ }^{18}$ : „Und in Gehorsam gegenüber den frommen Männern, die dem Diener Gottes gedient haben, habe ich mich bereitwilligst diesem kleinen Dienst des Wortes unterzogen, ..."19

\section{Grösse des Gegenstands (rerum magnitudo)}

Im Epilog der Vita des Gregorios Dekapolites (BHG 711) macht der Verfasser folgende Feststellung hinsichtlich der Größe des Gegenstands ${ }^{20}$ : „...; denn selbst wenn ich, wie es in der Dichtung heißt, zehn Zungen hätte und zehn Kehlen, so würden sie keinesfalls genügen, um den Mann zu preisen."

Im Epilog der Vita des Michael Synkellos (BHG 1296) heißt es im Rahmen der Anrufung des Vaters durch den Verfasser ${ }^{21}$ : ,Jenseits der menschlichen Möglichkeiten ist nämlich der Bericht über dein Bekenntnis und dein Leben, deren Vollbringung außergewöhnlich und deren Lobpreis einem Menschen nicht möglich ist."

Fast wortgleich findet sich dieser Topos bereits im Epilog der Vita des Stephanos des Jüngeren (BHG 1666) ${ }^{22}$ : „Jenseits des menschlichen Verständnisses ist nämlich dein Martyrium, dessen Vollbringung außergewöhnlich und dessen Lobpreis einem Menschen nicht möglich ist."

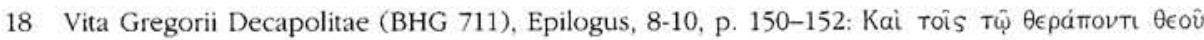

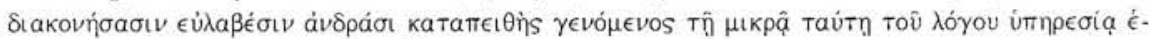

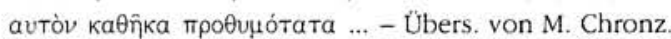

19 Weitere Beispiele: Vita Georg. Amastr. (BHG 668) cap. 48, p. 70,9-14; Vita Stephani iun. (BHG 1666) cap. 77, p. 175,26f. (Epiphanios); Vita Germani patriarchae (BHG 697) cap. 32, p. 240 (anonym); Vita Euthymii iun. (BHG 655) cap. 39, p. 204,10f.

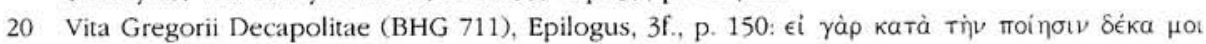

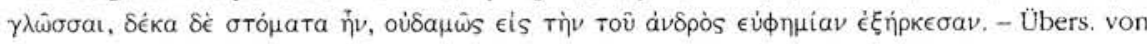
M. Chronz. Vgl. Hom., Il. 2,489.

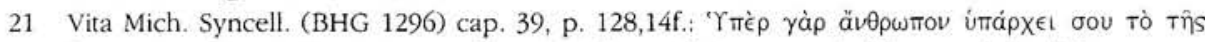

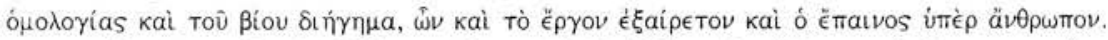

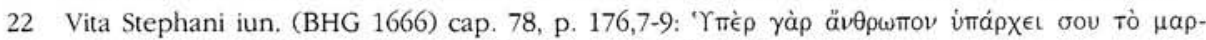

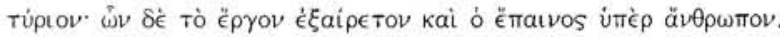


Der Epilog der Vita der Athanasia von Aigina (BHG 180) beginnt folgendermaßen $^{23}$ : „Soviel nur, wie allein zur Erinnerung euch gottliebenden (sc. Zuhörern) gereicht, ist in Maßen von mir hier jetzt erzählt worden. Wort für Wort aber alles zu erzählen, ist nicht nur mir allein unmöglich, sondern auch denen, die mich im Hinblick auf das Leben ${ }^{24}$ weit überragen und eine dem angemessene Redegabe besitzen."

Im Epilog der Vita des Georgios von Amastris (BHG 668) heißt $\mathrm{es}^{25}$, die Dürftigkeit der Worte lasse die Größe der im Enkomion Besungenen um so deutlicher hervortreten

Im Epilog der Vita des Patriarchen Germanos (BHG 697) wird die Größe des Gegenstands folgendermaßen impliziert ${ }^{26}:$ „Möchtest du doch geneigten Ohres diese mühsam geschriebene Erzählung gnädig annehmen, auch wenn sie weniger darstellt, als es angemessen ist." 27

\section{Weniges von Vielem (expluribus pauca)}

Im Epilog der Vita des Nikon „Metanoeite“ (BHG 1366-67) gibt der Verfasser folgendermaßen kund, daß er nur einige der vielen großen Werke des Heiligen berichten $\mathrm{kann}^{28}$ : „Leichter ist es nämlich, die Vielzahl der Sterne zu zählen und den Sand am Strand oder mit einem Becher die Menge des Meerwassers zu messen oder auch irgendeine andere der endlosen Aufgaben zu erledigen, als alle seine Werke zu erwähnen und zu erzählen, die durch keine Rede wiederzugeben und durch kein Gehör aufzunehmen sind wegen ihrer großen Menge."

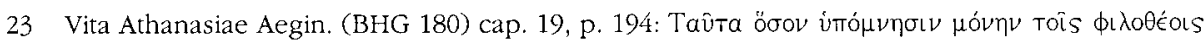

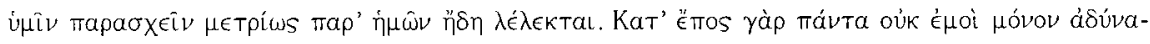

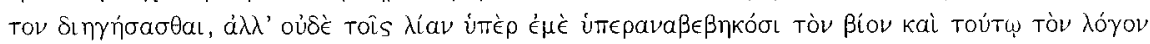

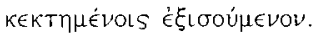

24 Gemeint ist wohl das Lebensalter, vgl. die Übers. von A. Laiou, in: Holy Women 157, oder auch die Lebensführung

25 Vita Georg. Amastr. (BHG 668) cap. 48, p. 70,15f.

26 Vita Germani patriarchae (BHG 697) cap. 32, p. 240. - Übers. von L. Lamza.

27 Weitere Beispiele: Vita Petri Atroënsis (BHG 2364) cap. 86, p. 223,36-225,5; Vita Macarii Pelecetae (BHG 1003) cap. 23, p. 163,21f.; Vita Euphrosynae iun. (BHG 627) cap. 48, p. 877E; Vita Niconis (BHG 1366-67) cap. $77,1-8$, p. 266-268; Vita Steph. Sabait. (BHG 1670) cap. 188, p. $584 \mathrm{~B}$.

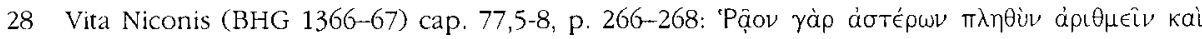

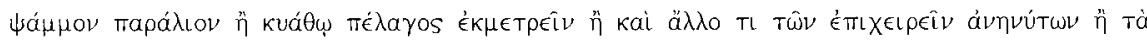

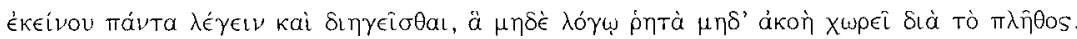


Im Epilog der Vita des Styliten Alypios (BHG 65) bemerkt der Verfasser im Zuge seiner Anrufung des Vaters ${ }^{29}$ : „Aus vielen wählte ich nur wenige deiner Taten aus, von denen ich bei den meisten auch zugegen war.“

Der Epilog der Vita der Athanasia von Aigina (BHG 180) beginnt folgendermaßen $^{30}$ : „Soviel nur, wie allein zur Erinnerung euch gottliebenden (sc. Zuhörern) gereicht, ist in Maßen von mir hier jetzt erzählt worden." Das impliziert natürlich, daß es noch viel mehr zu sagen gäbe ${ }^{31}$. Anschließend geht der Verfasser über zur „Größe des Gegenstands": Er hätte freilich niemals alles sagen können

Im Epilog der Vita des Petros von Atroa (BHG 2364) stellt der Verfasser folgende rhetorische Frage ${ }^{32}$ : „Wer könnte erzählen oder welche Zunge verkünden die an dem heilbringenden und glücksspendenden Grab selbst sich Tag für Tag ereignenden und sich jeden Tag vollendenden Zeichen und Wunder, immerwährend und reichlich bis heute?" Damit verweist der Verfasser auf den Umstand, daß in seiner Vita nur Weniges von Vielem zur Sprache gekommen ist ${ }^{33}$.

\section{E. Zweifelsfrage}

Eine Zweifelsfrage findet sich etwa im Epilog der Vita des Michael Synkellos (BHG 1296) ${ }^{34}$ : „Was soll ich nun zu dir sagen, o allerheiligster vater und heiliger Michael?"

Ganz ähnlich wiederum bereits in der Vita des Stephanos des Jüngeren $\left(\right.$ BHG 1666) ${ }^{35}$ : „Was soll ich nun auch zu dir sagen, o Vater, heiliger Märtyrer und Sieggekrönter über die Sieggekrönten, deines Namens würdiger Stephanos?"

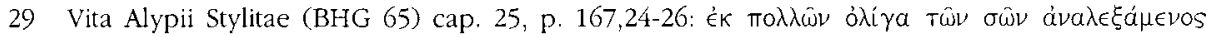

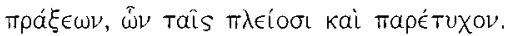

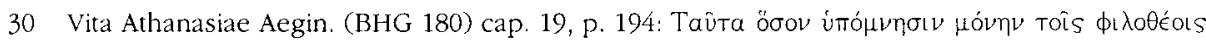

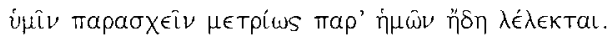

31 Die Vita ist im übrigen tatsächlich recht kurz und knapp.

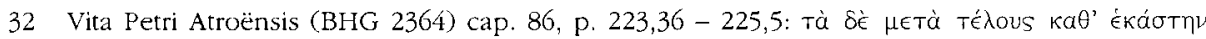

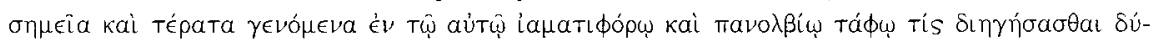

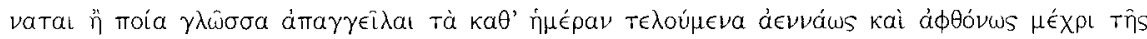
$\delta \in \overline{\mathrm{u} p o}$;

33 Weitere Beispiele: Vita Eliae iun. (BHG 580) cap. 76, p. 120, 1648-1655; Vita Germani patriarchae (BHG 697) cap. 32, p. 240; Vita Stephani iun. (BHG 1666) cap. 77, p. 176,2f.

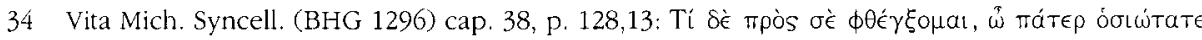
кai ä

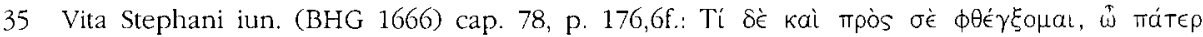

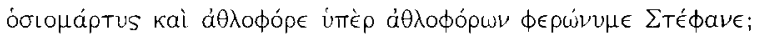


Im Epilog des „süditalienischen“ Schlusses ${ }^{36}$ der Vita des Gregorios von Agrigent (BHG 707) stellt der Verfasser ${ }^{37}$ die Frage ${ }^{38}$ : „Aber was soll ich weitschweifig den göttlichen Gregorios mit den gottgelehrten Vätern vergleichen?"

Eingangs des Epilogs der Vita des Styliten Alypios (BHG 65) fragt der Au$\operatorname{tor}^{39}$ : „Doch mit welchen Leuten sollte ich ihn etwa vergleichen, ohne daß er diese ganz klar übertreffen dürfte?"

\section{$\$ 2$. Begründungstopos (causa scribendi)}

\section{A. Mittel gegen das Vergessen (oblivionis remedium)}

Im Epilog der Vita des Gregorios Dekapolites (BHG 711) bekennt der Verfasser zunächst seine mangelnde Eignung für die Aufgabe und erklärt dann, er schreibe nur aus einem einzigen Grund, nämlich ${ }^{40}:$,..., nur um es denen, die Gott lieben, im Gedächtnis zu halten."

Im Epilog der Vita des Nikon „Metanoeite“ (BHG 1366-67) gibt der Verfasser seinen Grund für das Schreiben folgendermaßen $a n^{41}$ : ,..., damit nun nicht etwa die durch die Kraft des dir innewohnenden Heiligen Geistes vollbrachten Wundertaten den späteren Zeiten entgingen und so durch die Zeit ausgelöscht würden, und damit auch noch denen, die deine Zuneigung und Vertrautheit mit Gott nicht mit eigenen Augen gesehen haben, diese offenbar sei, ist dies von uns geschrieben worden."

Im Epilog der älteren Version der Vita des Ioannikios (BHG 936; Petros) versichert der Verfasser Petros im Rahmen der Anrufung des Heiligen ${ }^{42}$; ,..., daß du von uns nicht vergessen wirst, auch wenn du von uns gegangen bist und dein

36 Vgl, dazu Berger, Gregorios 23. 93. 127.

$37 \mathrm{Zu}$ ihm vgl. Berger, Gregorios $48 \mathrm{f}$.

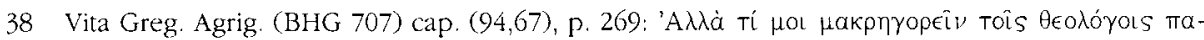

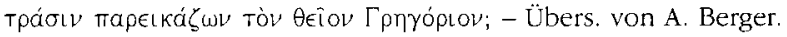

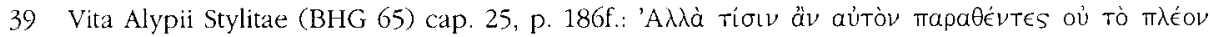

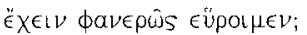

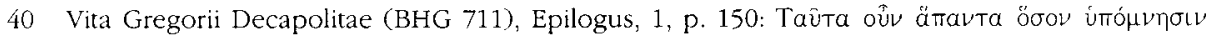

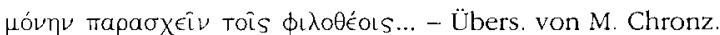

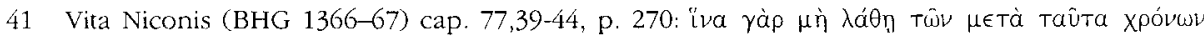

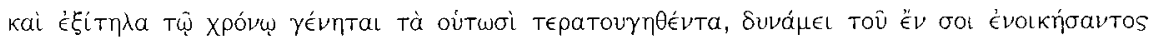

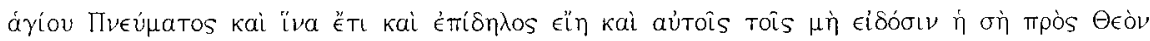

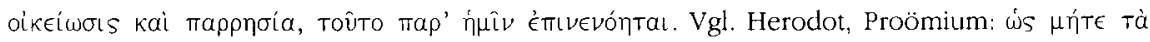

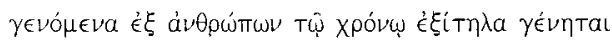

42 Vita Ioannicii (BHG 936; Petros) cap. 72, p. 435C. 
Leben vergangen ist nach den Gesetzen der Natur, doch die Erinnerung an dich wird auf ewig bei uns bleiben." Es wird vom Verfasser hier zwar nicht ausgesprochen, aber doch impliziert, daß dies auch und zuvörderst durch die Abfassung der Vita und die Pflege seines Heiligenkults geschieht.

\section{B. Erbaulicher Zweck (exemplum)}

Im Epilog der Vita des Gregorios Dekapolites (BHG 711) heißt es, nachdem der Verfasser erklärt hat, daß er den Auftrag zur Abfassung der Vita bereitwillig übernommen hat ${ }^{43}$ : „..., voller Zuversicht und in der zweifellosen Hoffnung auf Gott, daß das Gesagte den Hörern nutzbringend sein und viele zur Tapferkeit und zu ähnlichem Eifer im höchsten Grade anstacheln wird."

Ähnlich wie schon im Proöm besteht dieser Topos häufig aus einer Reihe von Beispielen von Vorgängern, die schließlich zum Vitenhelden selbst als erbauliches Vorbild hinführt. Ein typisches Beispiel dafür findet sich etwa in der Vita Konstantins des Juden (BHG 370) ${ }^{44}$ : Es wird zunächst der Prophet Elija angeführt, dessen Wortgewalt und Wundertätigkeit auch Konstantin geeignet habe. Ferner wird Johannes der Täufer als das größte Vorbild überhaupt unter den von Frauen Geborenen, also den Menschen, dargestellt. Er werde nur noch übertroffen durch Christus selbst. Der heilige Antonios, der Wüstenvater, habe die Wüste durch sein engelgleiches Leben geheiligt. Der heilige Sabas und die anderen Asketen hätten durch ihr tugendhaftes Leben die ewige Seligkeit erlangt. Schließlich heißt es über Konstantin ${ }^{45}$ : „Dieser berühmte Mann nun sammelte in der Art einer Biene von allen das Beste zusammen. Er war bestrebt, hinter keinem von ihnen als Zweiter zurückzustehen, sondern wie durch einen Spiegel in sich selbst aller Leben aufzuzeigen und aller Gnaden sowohl durch sein Leben als auch durch seine Wunder in dem unnachahmlichen Charakter der eigenen Lebensführung aufzuweisen." 46

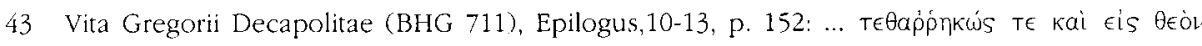

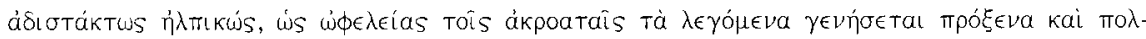

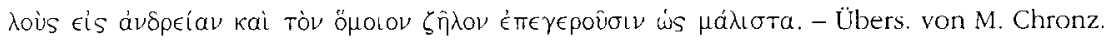

44 Vita Constantini Iudaei (BHG 370) cap 86, p. 655C-D.

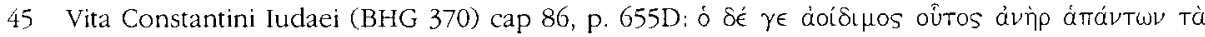

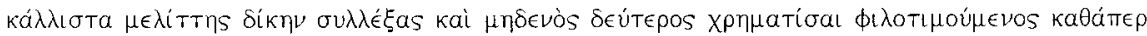

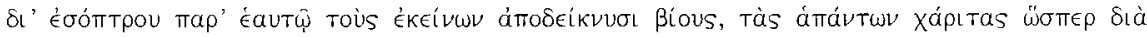

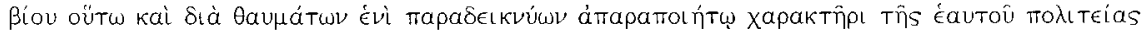




\section{Wissensbesitz verpflichtet zur Mitteilung (talentum)}

Im Epilog der Vita des Nikon "Metanoeite" (BHG 1366-67) macht der Verfasser die Feststellung, daß ein Übergehen der Werke des Nikon ein Verlust wäre. Folglich fühlte er sich gezwungen, sie zumindest teilweise zu erzählen ${ }^{47}$ : „Aber das unmäßige Ausdehnen der Rede und das detaillierte Aufzählen der durch ihn bewirkten und herbeigeführten Wunder ist gleichermaßen unpassend wie unmöglich und keine einfach zu erbringende Leistung, wie es auch wiederum einfach schädlich wäre, alles zu übergehen." Dieser Topos ist in den Epilogen eher selten und läßt sich nur gelegentlich finden. Die Ursache dafür könnte sein, daß an dieser Stelle der Vita, nämlich im Epilog, ja bereits alles mitgeteilt worden ist.

\section{Autorität der Alten (auctoritas antiquorum)}

Im Epilog der Vita des Michael Synkellos (BHG 1296) beruft sich der Verfasser folgendermaßen auf die „Autorität der Alten“ in Gestalt älterer Gewährsleute ${ }^{48}$ : „Diese Dinge wurden durch mich für das vorliegende bescheidene Werk in Erfahrung gebracht von den Verwandten des Heiligen, seinen Bekannten, Tischgenossen, Gefährten und Schülern, und überdies auch von den Verbündeten des Tyrannen, die noch in der Blüte dieses Lebens stehen." Der Toposcharakter dieser Angaben ist im Falle dieser Vita besonders offensichtlich, da sich dieser Abschnitt in fast wortwörtlicher Übereinstimmung auch in der Vita Stephanos' des Jüngeren (BHG 1666) findet ${ }^{49}$, die dem Autor der Vita des Michael Synkellos nicht nur in diesem Punkt als Vorlage diente ${ }^{50}$.

46 Weitere Beispiele: Vita Ioannicii (BHG 936; Petros) cap. 72, p. 435A-B; Vita Steph. Sabaït. (BHG 1670) cap. $185-187$, p. $583 \mathrm{E}-584 \mathrm{~B}$ (die Tugenden des Stephanos werden als erbauliches und nachahmenswertes Beispiel vorgeführt); Vita Eliae iun. (BHG 580) cap. 76, p. 120, 1656-1659; Vita Germani patriarchae (BHG 697) cap. 32, p. 238-240; Vita Basilii iun. (BHG 263) cap. 59, p. *32B; Vita Euphrosynae iun. (BHG 627) cap. 49, p. 877E-F; Vita Danielis Stylitae (BHG 489) cap. 56, p. 147,23-33; Vita Lazari Galesiotae (BHG 979) cap. 255, p. 588E.

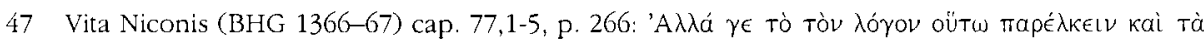

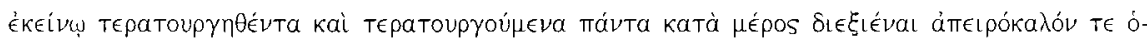

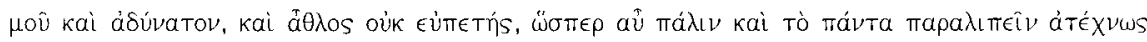
$\epsilon \pi \zeta \zeta$ ń

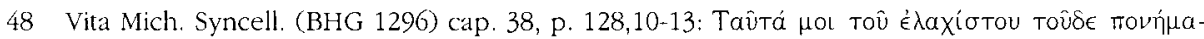

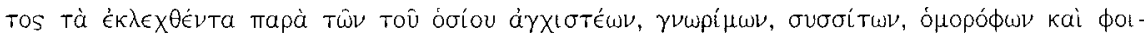

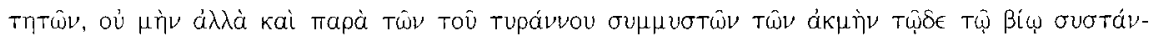
TwL.

49 Vita Stephani iun. (BHG 1666) cap. 77, p. 175,22-25. 
Im Epilog der Vita der Athanasia von Aigina (BHG 180) heißt es ${ }^{51}$ : „Ich bin keineswegs irgendweichen Erfindungen gefolgt, sondern habe nur den Dingen vertraut, die ich selbst mit eigenen Augen gesehen habe, sowie vertrauenswürdigen Frauen (Nonnen), die nur die Wahrheit sagen und mit unserer gesegneten Mutter ihr ganzes Leben in herausragender Weise zusammenlebten und über sie gut Bescheid wußten."

Auch im Epilog der Vita des Paulos des Jüngeren (BHG 1474) wird betont, daß der Verfasser sich nicht etwa auf irgendwelche zufälligen Gewährsleute verlassen habe, sondern nur auf die „wahrheitsliebenden und trefflichsten Hüter der Tugend" 52 .

\section{$\$ 3$. Formeln (formulae)}

\section{A. Brevitas-Formel}

An einer Stelle des Epilogs ${ }^{53}$ der Vita des Nikon „Metanoeite“ (BHG 1366-67) bemerkt der Verfasser ${ }^{54}$ : „Aber das unmäßige Ausdehnen der Rede (...) ist gleichermaßen unpassend wie unmöglich ..." An anderer Stelle ${ }^{55}$ erklärt er, er wolle, aufgrund seiner Unfähigkeit, seiner Rede nun hier ein Ende setzen: Té $\lambda \circ \mathrm{S}$

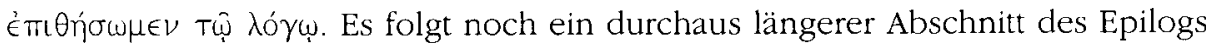
(39 Zeilen der Edition umfassend).

Im Epilog der älteren Version der Vita des Ioannikios (BHG 936) ${ }^{56}$ behauptet der Verfasser, der Mönch Petros, daß er dem Heiligen eine sehr kurze Grab-

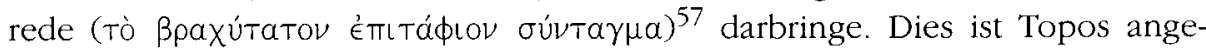
sichts der umfänglichen Vita, die sich über 51 Editionsseiten der AASS und

50 Vgl. den kritischen Apparat zur Edition der Vita von M.-F. Auzépy, wo die Parallelstellen jeweils vermerkt sind.

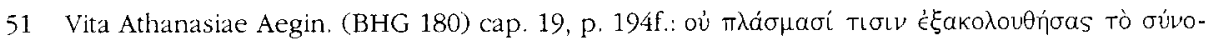

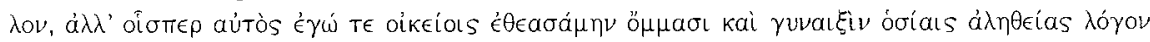

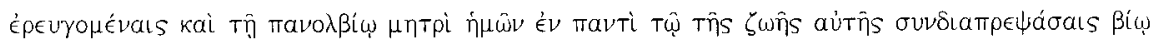

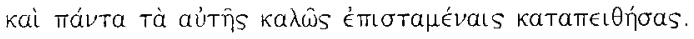

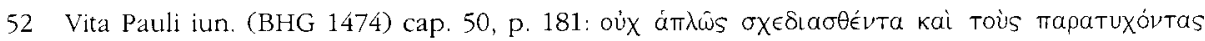

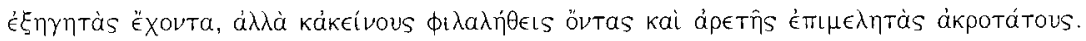

53 Zur Brevitas-Formel im Epilog vgl. Müller, Vita 588f. mit Anm. 20.

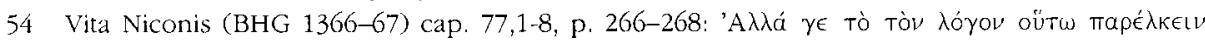

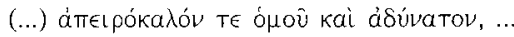

55 Vita Niconis (BHG 1366-67) cap. 77,11f., p. 268.

56 Vita Ioannicii (BHG 936; Petros) cap. 72, p. 435C.

57 Vgl. dazu D. F. Sullivan, in: Byzantine Defenders 350 Anm. 588. 
insgesamt 72 Kapitel (cap. 1-3: Prooimion, cap. 4-71: Narratio, cap. 72: Epilog) erstreckt

Im Epilog der Vita des Elias des Jüngeren (BHG 580) bemerkt der Verfasser, er habe nun nicht alles über den Heiligen mitgteilt ${ }^{58}, \ldots$ damit nicht irgendwann einer, der viele Dinge gehört hat, sich über die Unmäßigkeit der Erzählung beschwere."

Im Epilog der Vita des Michael Synkellos (BHG 1296) bemerkt der Verfasser an einer Stelle ${ }^{59}:$,..., um es kurz zu sagen, ...“

\section{B. Veritas-Formel}

Im Epilog der Vita des Gregorios Dekapolites (BHG 711) erklärt der Verfasser folgendes bezüglich seiner Gewährsleute ${ }^{60}:$,.., wobei ich weder den Märchen alter Weiber noch trügerischen Gerüchten oder Gespinsten der Einbildung in irgendeiner Weise nachgegeben, vielmehr Mündern vertraut habe, die wahre Worte von sich geben."

Im Epilog der Vita des Basileios des Jüngeren (BHG 180) wird folgendes gesagt $^{61}$ : „Und bei der Wahrheit, liebe Brüder in Christo, so verhalten sich die Dinge."

Im Epilog der Vita des Paulos des Jüngeren (BHG 1474) wird betont ${ }^{62}$, daß der Verfasser sich nur auf „wahrheitsliebende (sc. Gewährsleute) und trefflichste Hüter der Tugend" verlassen habe.

Stephanos Diakonos behauptet im Epilog der Vita des Stephanos des Jünge-

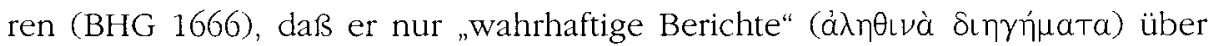
den Heiligen in seine Vita aufgenommen habe ${ }^{63}$.

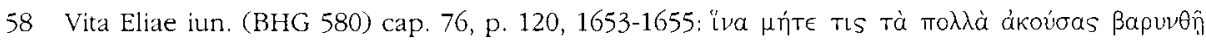

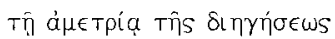

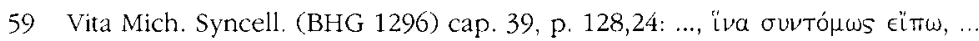

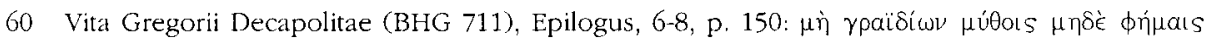

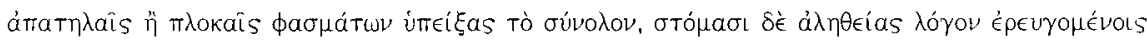

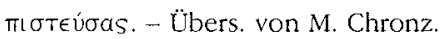

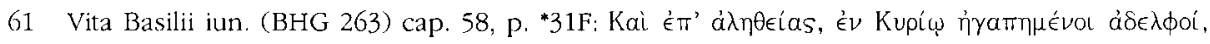

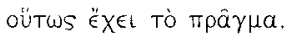

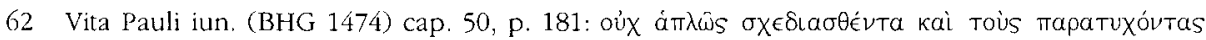

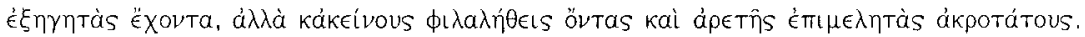

63 Vita Stephani iun. (BHG 1666) cap. 77, p. 175,22-25. - Weiteres Beispiel: Vita Athanasiae Aegin. (BHG 180) cap. 19, p. 194 f. 


\section{Mönchsformel}

Im Epilog der älteren Version der Vita des Ioannikios (BHG 936; Petros) bittet der Verfasser, der Mönch Petros, den Heiligen darum, er möge seiner gedenken, des „nutzlosen Dieners" (

Im Epilog der Vita des Makarios vom Peleketeklosters (BHG 1003) bittet der Verfasser den heiligen Vater, er möge doch die Fehler „seiner Schwäche“ bzw. „Kraftlosigkeit" korrigieren. Er nennt sich ferner einen „unwürdigen Nachfolger" und spricht von sich als "seiner Schlichtheit" 65 .

Stephanos Diakonos bezeichnet sich am Beginn des Epilogs der Vita des Stephanos des Jüngeren (BHG 1666) als „Geringsten“66. Ganz wortgleich wiederum diese Formel in der Vita des Michael Synkellos (BHG 1296) ${ }^{67}$. Gegen Ende des Epilogs bezeichnet Stephanos Diakonos sich als „unwürdig“ (ó ảv$\alpha \dot{\xi}(\mathrm{LS})^{68}$

\section{$\$ 4$. Anrufung (epiklesis, invocatio)}

In den Epilogen findet sich an Stelle der in den Prooimia häufigen formelhaften Anrufung Gottes, der dem Verfasser „den Mund öffnen möge“, sehr häufig eine Invocatio des Heiligen selbst, der für den Verfasser und die Zuhörer Fürsprache bei Jesus Christus einlegen möge. Diese Anrufung überschreitet im Epilog den Rahmen einer Formel und hat einen größeren Umfang; überhaupt spielt sie in den Epilogen eine herausragende Rolle und hat deshalb einen eigenen Paragraphen erhalten.

Im Epilog der Vita des Gregorios Dekapolites (BHG 711) wird der Heilige (sc. Gregorios Dekapolites) folgendermaßen angerufen ${ }^{69}$ : „Du aber, Glückseliger und Genosse derer, die in Heiligkeit und Gerechtigkeit aufstrahlen, stelle dich durch deine Fürbitten zum Allmächtigen vor deine Herde und deine Schutzbefohlenen. Wende jede Versuchung und jedes Ungemach von deiner Herde ab und erbitte, daß der Kirche beständiger Friede gegeben werde. Vertreibe mit dem Stab deines Gebetes die Häresien samt jener, die sich gerade jetzt

64 Vita Ioannicii (BHG 936; Petros) cap. 72, p. 435C.

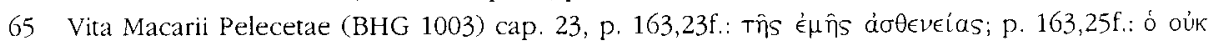

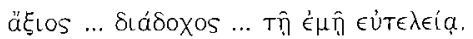

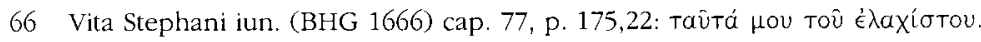

67 Vita Mich. Syncell. (BHG 1296) cap. 39, p. 128,10.

68 Vita Stephani iun. (BHG 1666) cap. 78, p. 177,16. - Weiteres Beispiel: Vita Niconis (BHG 1366-

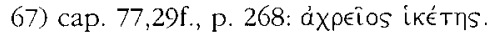


frech brüstet, wie dumme, tückisch beißende Hunde. Denn da du im Lichte des Herrn und in den unvergänglichen Wohnungen und Zelten herbergst und die Lampe der Keuschheit durch das Öl der guten Taten der Askese unverlöscht bewahrt hast und mit denen, die Gott von Ewigkeit her wohlgefällig sind, die Güter genießt, die kein Auge gesehen, die kein Ohr gehört hat und die in das Herz keines Menschen gedrungen sind, vermagst du alles in dem, der dich stärkt, Christus unserem Herrn, dem alle Herrlichkeit gebührt, alle Ehre und Anbetung, zugleich mit dem Vater und dem Heiligen Geist, jetzt und immerdar und in alle Ewigkeit."

Im Epilog der Vita des Lazaros von Galesion (BHG 979) wird der Heilige nicht direkt um Fürsprache angerufen, denn diese wird gewissermaßen vorausgesetzt. Es wird folglich einfach festgestellt, daß der heilige Vater jetzt im Himmel sei und dort Fürsprache für die Menschen einlege ${ }^{70}$ : „Für uns, die wir ihn auf Erden preisen, legt er inbrünstig und ohne Unterlaß Fürsprache ein, auf daß wir von Gott Vergebung erhalten mögen für die uns anhaftenden Schlechtigkeiten und die von uns begangenen Sünden."

Im Epilog der Vita des Nikon „Metanoeite“ (BHG 1366-67) findet sich wieder eine längere, echte Anrufung des Heiligen durch den Verfasser ${ }^{71}$ : „Diese

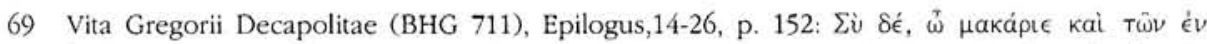

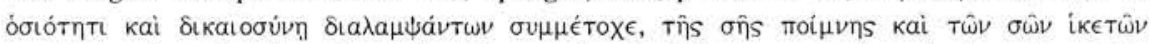

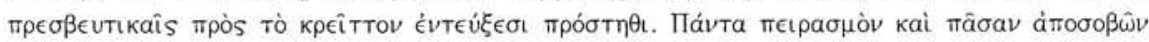

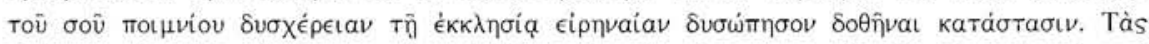

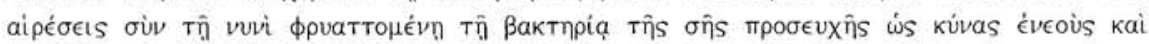

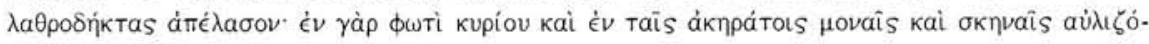

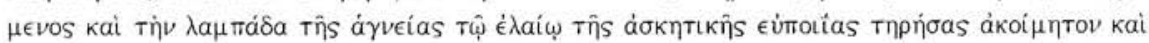

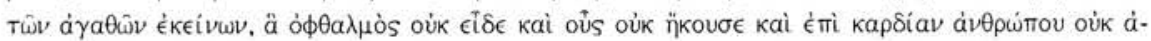

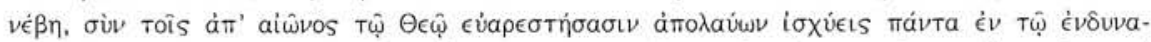

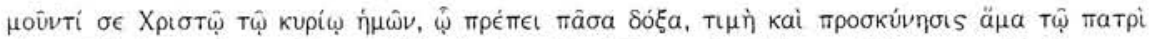

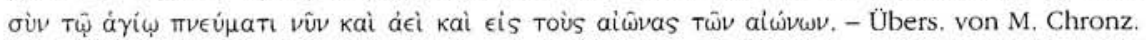

70 Vita Lazari Galesiotae (BHG 979) cap. 255, p. 588: ن்ாغ்

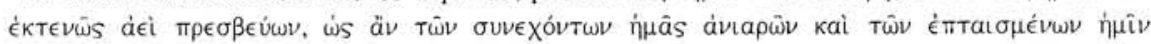

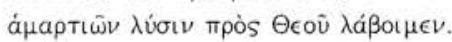

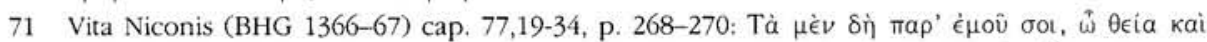

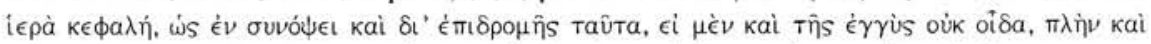

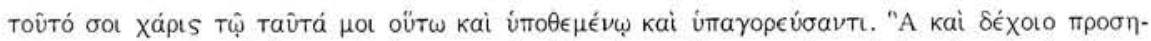

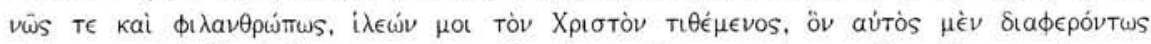

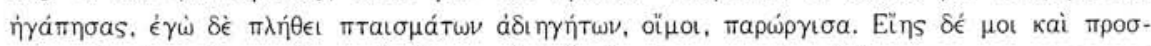

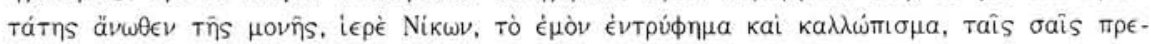

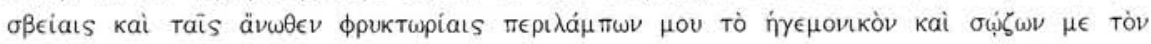

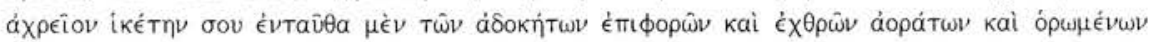

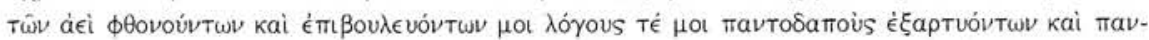

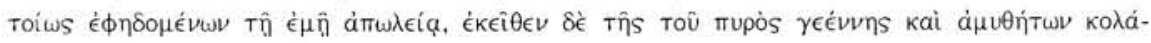
$\sigma \epsilon \omega \nu$. 
Dinge nun sind von mir für dich, o göttliches und heiliges Haupt, in Kürze und Eile niedergeschrieben; ob sie deiner auch nur annähernd würdig sind, weiß ich nicht. Nun aber mögen sie dir ein Geschenk sein, der du mir diese Dinge so aufgetragen und diktiert hast. Mögest du sie nun auch wohlwollend und menschenfreundlich annehmen und mir Christus geneigt machen, den du selbst auBerordentlich geliebt hast, den ich aber durch die große Zahl meiner unaussprechlichen Fehltritte, wehe mir, erzürnt habe. Mögest du mir sowohl Vorsteher sein von oberhalb des Klosters, heiliger Nikon, als auch meine Freude und mein Stolz! Durch deine Fürsprachen und durch deine Feuerzeichen von oben her ${ }^{72}$ beleuchte meine Abtswürde und errette mich, deinen unwürdigen Bittsteller hienieden vor unvorhersehbaren Angriffen und Feinden, unsichtbaren und sichtbaren, die stets von Neid erfüllt sind und Pläne gegen mich schmieden und sowohl mit jeder Art von Worten gegen mich rüsten als auch sich auf jede Art freuen über mein Verderben! Dann errette mich vor dem Verderben des Höllenfeuers und den unsagbaren Höllenqualen!“73

Sofern kein Textabbruch vorliegt, münden die Epiloge bzw. die Viten ${ }^{74}$ gewöhnlich in Gebetsformeln - am häufigsten sind allgemeine Gebetsformeln, Auszüge aus dem „Vaterunser" und ähnliches - und endigen mit dem das Gebet beschließenden „Amen“.

72 Vgl oben Kap. 12: „Zeichen“, \& 1. „Licht”, S. 213-216.

73 Weitere Beispiele: Vita Nicephori patriarchae (BHG 1335) p. 215,13-217,36; Vita Theod. Cyth. (BHG 2430) p. 289,257 - 291,330; Vita Mariae iun. (BHG 1164) cap. 33, p. 705A-E; Vita Athanasiae Aegin. (BHG 180) cap. 20, p. 195; Vita Ioannicii (BHG 936; Petros) cap. 72, p. 435C-F; Vita Constantini Iudaei (BHG 370) cap. 87, p. 655E-656D; Vita Euthymii iun. (BHG 655) cap. 39, p. 204f.; Vita Pauli iun. (BHG 1474) cap. 50, p. 181; Vita Georg. Amastr. (BHG 668) cap. 48, p. 70f; Vita Steph. Sabaït. (BHG 1670) cap. 188, p. 584B; Vita Lucae iun. (BHG 994b) cap. 100, p. 223; Vita Germani patriarchae (BHG 697) cap. 32, p. 238-240; Vita Mich. Syncell. (BHG 1296) cap. 39, p. 128,15-31; Vita Stephani iun. (BHG 1666) cap. 78, p. 176,14-177,26; Vita Euthymii iun. (BHG 655) cap. 39, p. 204f.; Vita Ioannis Damasceni (BHG 884) cap. 39, col. 489AB; Vita Theod. Stud. (BHG 1755) cap. 130, col. 229D - 232A. - Vgl auch Gamillscheg, Gegebenheiten $1-13$

74 S. dazu bereits oben S. 340 Anm. 1. 
